\title{
Testing quantised inertia on the emdrive
}

\author{
M.E. McCulloch*
}

April 13, 2016

\begin{abstract}
It has been shown that truncated cone-shaped cavities with microwaves resonating within them move slightly towards their narrow ends (the emdrive). Standard physics has no explanation for this and an error has not yet been found. It is shown here that this effect can be predicted by assuming that the inertial mass of the photons in the cavity is caused by Unruh radiation, whose wavelengths must fit exactly within the cavity, using a theory already applied successfully to astrophysical anomalies such as galaxy rotation where the Unruh waves have to fit within the Hubble scale. In the emdrive this means that more Unruh waves are allowed at the wide end, leading to a greater inertial mass for the photons there, and to conserve momentum the cavity must move towards its narrow end, as observed. The model predicts thrusts of: $3.8,149,7.3,0.23,0.57,0.11$, 0.64 and $0.02 \mathrm{mN}$ compared with the observed thrusts of: 16, 147, 9, $0.09,0.05,0.06,0.03$, and $0.02 \mathrm{mN}$ and predicts that if the axial length is equal to the diameter of the small end of the cavity, the thrust should be reversed.
\end{abstract}

\section{Introduction}

It was first demonstrated by Shawyer (2008) that when microwaves resonate within a truncated cone-shaped cavity a small, unexplained thrust and acceleration occurs towards the narrow end. In one example when $850 \mathrm{~W}$ of power was put into such a cavity with end diameters of $16 \mathrm{~cm}$ and $12 \mathrm{~cm}$ and a $\mathrm{Q}$ value (dissipation constant) of 5900 the thrust measured was $16 \mathrm{mN}$ towards the narrow end. The results from two of Shawyer's experiments are shown in Table 1 (rows 1-2). There is no explanation for this behaviour in standard physics because it violates the conservation of momentum, and Shawyer's own attempt to explain it using special relativity is not convincing, as this theory also should obey the conservation of momentum (Mullins, 2006).

Nethertheless, this anomaly has been confirmed by a Chinese team (Juan et al., 2012) who put $80-2500 \mathrm{~W}$ of power into a similar cavity at a frequency of

*SMSE, Plymouth University, Plymouth, PL4 8AA. mike.mcculloch@plymouth.ac.uk 
$2.45 \mathrm{GHz}$ and measured a thrust of between $70 \mathrm{mN}$ and $720 \mathrm{mN}$. Their result cannot however be used for testing here since they did not specify their cavity's quality factor or their geometry. A similar anomaly was seen by Fetta (2012) who claimed the effect was due to grooves in one of the ends of the cavity and not the asymmetry of the cone. A later test by NASA showed the grooves made no difference to the thrust.

Further confirmation of the emdrive was obtained by a NASA team (Brady et al., 2014), most recently in a vacuum proving that the effect is not due to moving air. Their four results are shown in Table 1 (rows 4 to 7 ). They did provide details of their $\mathrm{Q}$ factor and some details of their cavity's geometry.

McCulloch (2007) has proposed a new model for inertia (MiHsC) that assumes that the inertia of an object is due to the Unruh radiation it sees when it accelerates, radiation which is also subject to a Hubble-scale Casimir effect. In this model only Unruh wavelengths that fit exactly into twice the Hubble diameter are allowed, so that a greater proportion of the waves are disallowed for low accelerations (which see longer Unruh waves) leading to a gradual new loss of inertia as accelerations become tiny. MiHsC modifies the standard inertial mass $(m)$ to a modified one $\left(m_{i}\right)$ as follows:

$$
m_{i}=m\left(1-\frac{2 c^{2}}{|a| \Theta}\right)=m\left(1-\frac{\lambda}{4 \Theta}\right)
$$

where $\mathrm{c}$ is the speed of light, $\Theta$ is twice the Hubble distance, ' $|\mathrm{a}|$ ' is the magnitude of the relative acceleration of the object relative to surrounding matter and $\lambda$ is the peak wavelength of the Unruh radiation it sees. Eq. 1 predicts that for terrestrial accelerations (eg: $9.8 \mathrm{~m} / \mathrm{s}^{2}$ ) the second term in the bracket is tiny and standard inertia is recovered, but in low acceleration environments, for example at the edges of galaxies (when a is small and $\lambda$ is large) the second term in the bracket becomes larger and the inertial mass decreases in a new way so that MiHsC can explain galaxy rotation without the need for dark matter (McCulloch, 2012) and cosmic acceleration without the need for dark energy (McCulloch, 2007, 2010). However, astrophysical tests like these can be ambiguous, since more flexible theories like dark matter can be manipulated to fit the data, and so a controlled laboratory test like the EmDrive is preferable.

The difficulty of demonstrating MiHsC on Earth is the huge size of $\Theta$ in Eq. 1 which makes the effect very small unless the acceleration is tiny, as in deep space. One way to make the effect more obvious is to reduce the distance to the horizon $\Theta$ and this is what the emdrive may be doing since the radiation within it is accelerating so fast that the Unruh waves it sees will be short enough to be limited by the cavity walls in a MiHsC-like manner. McCulloch (2015) showed that assuming that the inertial mass of the photons is determined by $\mathrm{MiHsC}$ and the width of the cavity, and assuming the conservation of momentum, predicts a new force of size 


$$
F=-\frac{P Q L}{c}\left(\frac{1}{w_{s}}-\frac{1}{w_{b}}\right)
$$

where $\mathrm{P}$ is the power input, $\mathrm{Q}$ is the quality factor, $\mathrm{L}$ is the axial length, $\mathrm{c}$ is the speed of light and $w_{s}$ and $w_{b}$ are the widths of the small and big ends respectively. This formula predicts the observed emdrive thrusts quite well (see the Table, column 6) however it assumed that the Unruh waves only resonate perpendicular to the axis of symmetry and so predicts infinite thrust for a pointed cone $\left(w_{s}=0\right)$. In this paper this model is modified to approximate the microwaves' resonance in three-dimensions.

\section{Method}

The so-called emdrive is a microwave resonant cavity shaped like a truncated cone, with one round end larger than the other (see Figure 1). When an electromagnetic field resonates in the cavity we can consider the conservation of momentum for the photons as they move along the axis

$$
\frac{\partial(m v)}{\partial t}=0=m \frac{\partial v}{\partial t}+v \frac{\partial m}{\partial t}
$$

The first term on the right hand side is the force (mass times acceleration) that must be exerted on the photons to change their speed to conserve their momentum if MiHsC changes their mass. This force, $\mathrm{F}$, is then, from Eq. 3:

$$
F=-c \frac{\partial m}{\partial t}
$$

So that

$$
F=-c \frac{\partial m}{\partial x} \frac{\partial x}{\partial t}=-c^{2} \frac{\partial m}{\partial x}
$$

Normally, of course, photons are not supposed to have inertial mass in this way, but here this is assumed. It is not clear what the size of this mass is, but it is clear for example that light inside a mirrored box produces a kind of inertial mass for the box. It is also assumed that the inertial mass of the microwave photons (whatever its absolute value) is affected by $\mathrm{MiHsC}$, but instead of the horizon being the far-off and spherically symmetric Hubble horizon as before, the horizon is now made by the asymmetric walls of the cavity. This is possible because the photons involved are travelling at the speed of light and are bouncing very fast between the two ends of seperation 'L' and their acceleration $\left(a \sim v^{2} / L\right)$ is so large that the Unruh waves that are assumed in MiHsC to produce their inertial 
mass are about the same size as the cavity, so they can be affected by its walls, unlike the Unruh waves for a normal acceleration which would be far to long to be affected by the cavity. This dependence of the inertial mass on the width of the cavity means that the inertial mass, is corrected by a MiHsC-like factor (Eq. 1) so the force in Eq. 5 is modified as follows

$$
F=-c^{2}\left(\frac{m_{\text {bigend }}-m_{\text {smallend }}}{L}\right)
$$

where L is the axial length of the cavity. In McCulloch (2015) it was assumed that the waves only resonate perpendicular to the axis. This model was quite successful in predicting the emdrive thrusts (see the Table). MiHsC (Eq. 1) makes the assumption that the Unruh wavelengths are made up of a complete pseudo-Planckian spectrum, which is subsampled because some of the wavelengths do not fit within the cavity and so the energy in the spectrum decays linearly as the cavity narrows. Using Eq. 1, Eq. 6 becomes

$$
F=-\frac{m c^{2}}{L}\left(\frac{\lambda}{4 w_{s}}-\frac{\lambda}{4 w_{b}}\right)
$$

This formula is not valid for pointed emdrive cones where for example $w_{s}=0$, and to remedy this we need to consider resonance along the axis. To do this it is necessary to calculate the average distance between a photon at either end and the walls. We can do this in a simplified manner by looking at this distance along six orthogonal directions. Looking at Figure 1 and the distance from the centre of the left-hand end plate (denoted P) to the walls: these six directions are: to the left where the distance between $\mathrm{P}$ and the wall is zero, to the right where it is L, up and down and into and out of the page where it is $w_{s}$. This means that the (very approximate) average distance the Unruh waves have to resonate in at the narrow end are

$$
\bar{w}_{s}=\frac{0+L+4 w_{s}}{6}
$$

and at the wide end

$$
w_{b}=\frac{0+^{-} L+4 w_{b}}{6}
$$

Substituting these into Eq. 7 we get

$$
F=-\frac{3 m c^{2} \lambda}{2 L}\left(\frac{1}{L+4 w_{s}}-\frac{1}{L+4 w_{b}}\right)
$$


Here $\lambda$ is the wavelength of the Unruh radiation seen by the microwaves because they are being reflected back and forth by the cavity. This is given by $\lambda=$ $8 c^{2} / a=8 c^{2} /(2 c /(L / c))=4 L$ so that

$$
F=-6 m c^{2}\left(\frac{1}{L+4 w_{s}}-\frac{1}{L+4 w_{b}}\right)
$$

Using $E=m c^{2}$ and $E=\int P d t$ where $\mathrm{P}$ is the power input into the cavity, gives

$$
F=-6 \int P d t\left(\frac{1}{L+4 w_{s}}-\frac{1}{L+4 w_{b}}\right)
$$

Integrating $\mathrm{P}$ over one cycle (one trip of the microwaves from end to end) gives $\mathrm{Pt}$ where $\mathrm{t}$ is the time taken for the microwaves to travel from one end of the cavity's long axis to the other, which is $\mathrm{L} / \mathrm{c}$, so

$$
F=-\frac{6 P L}{c}\left(\frac{1}{L+4 w_{s}}-\frac{1}{L+4 w_{b}}\right)
$$

This is for one trip along the cavity, but the Q factor quantifies how many trips there are before the photon dissipates so we need to multiply by Q. Note that the force is towards the narrow end for both directions of travel as explained in Figure 1 and the discussion.

$$
F=-\frac{6 P Q L}{c}\left(\frac{1}{L+4 w_{s}}-\frac{1}{L+4 w_{b}}\right)
$$

Therefore MiHsC predicts that a new force will appear acting always towards the narrow end of the cavity. This formula has the advantage over that of McCulloch (2015) because it models, crudely, the resonance of the Unruh waves in three-dimensions.

\section{$3 \quad$ Results}

The table includes a summary of the various experimental results from Shawyer (2008) in rows 1 and 2 (denoted S1 and S2), the Cannae drive in row 3 (Fetta, 2012) denoted C1, and Brady et al. (2014) in rows 4 to 7 (denoted B1-B3 and $\mathrm{B} 4 \mathrm{v}$ is the recent vacuum test) and the vacuum test by Tajmar and Fiedler (2015) denoted T1. The Juan et al. (2012) data is excluded because they did not specify their $\mathrm{Q}$ factor or the geometry of their emdrive.

Column 1 names the experiment ( $\mathrm{S}$ for Shawyer's experiment, $\mathrm{C}$ for the Cannae drive, B for the NASA results and T for Tajmar and Fiedler). Column 2 shows the input power (in Watts). Column 3 shows the Q factor (dimensionless). 
Column 4 shows the axial length of the cavity. Column 5 shows the width of the big and small ends (metres). Column 6 shows the thrust predicted by onedimensional MiHsC (Eq. 2) (in milliNewtons), column 7 shows the prediction of the three-dimensional version of $\mathrm{MiHsC}$ derived in this paper (Eq. 14) and column 7 shows the thrust observed (milli-Newtons) in the experiment.

\begin{tabular}{|c|c|c|c|c|c|c|c|}
\hline Expt & $\mathrm{P}$ & $\mathrm{Q}$ & $\mathrm{L}$ & $w_{\text {big }} / w_{\text {small }}$ & $F_{1 d}$ & $F_{3 d}$ & $F_{\text {Obs }}$ \\
\hline \hline & $\mathrm{W}$ & & $\mathrm{m}$ & metres & $m N$ & $m N$ & $m N$ \\
\hline $\mathrm{S} 1$ & 850 & 5900 & 0.156 & $0.16 / 0.1275$ & 4.2 & 3.8 & 16 \\
\hline $\mathrm{S} 2$ & 1000 & 45,000 & 0.345 & $0.28 / 0.1289$ & 217 & 149 & $80-214$ \\
\hline $\mathrm{C} 1$ & 10.5 & $11 \times 10^{6}$ & 0.03 & $0.22 / 0.2$ & 5.3 & 7.3 & 9 \\
\hline $\mathrm{B} 1$ & 16.9 & 7,320 & 0.2286 & $0.2794 / 0.1588$ & 0.26 & 0.23 & 0.091 \\
\hline $\mathrm{B} 2$ & 16.7 & 18,100 & " & " & 0.63 & 0.57 & 0.05 \\
\hline $\mathrm{B} 3$ & 2.6 & 22,000 & " & " & 0.12 & 0.11 & 0.055 \\
\hline $\mathrm{B} 4 \mathrm{v}$ & 50 & 6730 & " & " & 0.70 & 0.64 & 0.03 \\
\hline $\mathrm{T} 1$ & 700 & 20 & 0.1008 & $0.1062 / 0.075$ & 0.02 & 0.02 & $0.02-0.11$ \\
\hline
\end{tabular}

Table 1. A summary of the fully documented emdrive experiments so far. Column 1 shows the experiment name, column 2 shows the input power, column 3 the Q factor, column 4 the cavity's axial length, column 5 shows the cavity end widths. Columns 6 and 7 show the thrusts predicted by the 1-d and 3-d versions of $\mathrm{MiHsC}$ respectively, and column 8 shows the observed thrust.

The Table shows that MiHsC predicts the experimental results quite well. The worst agreements are for $\mathrm{B} 2$ and $\mathrm{B} 4 \mathrm{v}$ where $\mathrm{MiHsC}$ is over a factor of ten out. In almost all cases MiHsC3d performs slightly better than MiHsC1d. The differences could be due to the approximate way MiHsC has been applied so far and it is unclear what the error bars on the observations are. The range of values for $\mathrm{S} 2$ gives some idea of the large uncertainty in the data. However, MiHsC predicts the correct order of magnitude for all the cases. This is encouraging given that this model is rather approximate (not fully three-dimensional), may be affected by uncertainties in the cavity geometry. It should also be noted that MiHsC has no adjustable parameters.

\section{Discussion}

To explain in a more intuitive manner: $\mathrm{MiHsC}$ predicts that as photons travel from the narrow end on the left of the emdrive to the wide end on the right (see the lower arrows in Figure 1) their inertial mass increases as more Unruh wavelengths fit at the righthand wide end of the cavity. In the figure this is shown by the arrow being thicker on the right. This change though, has violated the conservation of momentum, so we must now apply a force towards the narrow end to slow the photon down and conserve momentum. When the photons bounce off the wide end and move leftwards again towards the narrow end they lose inertial mass because fewer Unruh waves fit at the narrow end, so to conserve momentum it is now necessary to apply a force, again towards the narrow end, to speed the photons up. In both cases the new MiHsCian force 
is towards the narrow end and of a size, as shown in the Table, similar to the anomalous thrust that has been seen in the experiments.

More data is needed for comparison, and a more accurate modelling of the effects of MiHsC will be needed. This analysis for simplicity, assumed the microwaves only travelled along the axis and the three-dimensional resonance of the waves was only crudely modelled: a full 3 -d model is needed.

This proposal predicts the observations quite well, but makes two controversial assumptions. For example that the inertial mass of photons is finite (in defence of this, they do carry momentum) and varies in line with $\mathrm{MiHsC}$, and that the speed of the light is changing in the cavity. So it is important to suggest a definite test.

Both Eq. 14 and the simpler equation in McCulloch (2015), Eq. 2, predict that it should be possible to reverse the sign of the thrust by shortening the usual cavity length (L) or changing the frequency so that the Unruh waves fit better into the short end $\left(w_{s}\right)$ than the wide end $\left(w_{b}\right)$. This thrust reversal may have been seen in recent NASA experiments. Equation 14 also suggests that the anomalous force can be increased by increasing the power input, $\mathrm{P}$, or the quality factor of the cavity $(\mathrm{Q}$, the number of times the microwaves bounce between the two ends) and the speed of light on the denominator of Eq. 14 implies that if the value of $\mathrm{c}$ was decreased by a dielectric the effect would be enhanced.

\section{Conclusion}

More than eight tests in four independent labs have shown that when microwaves resonate within an asymmetric cavity an anomalous thrust is generated pushing the cavity towards its narrow end.

This force can be predicted fairly well by using a new model for inertia (MiHsC) which assumes that the inertial mass of the photons is caused by Unruh radiation whose wavelengths have to fit exactly inside the cavity so that the photons' inertial mass is greater at the wide end. To conserve momentum a new force appears to to push the cavity towards its narrow end, and the predicted force is similar to the thrust observed.

$\mathrm{MiHsC}$ suggests that the thrust can be increased by increasing the input power, the $\mathrm{Q}$ factor, or using a dielectric. As a direct test $\mathrm{MiHsC}$ predicts that the thrust can be reversed by making the length $L$ equal to the width of the narrow end.

\section{Acknowledgements}

Many thanks for Dr Jose Rodal and others on an NSF forum for estimating from photographs the proportions of the various experimental arrangements. 


\section{References}

Brady, D.A., H.G. White, P. March, J.T. Lawrence and F.J. Davies, 2014. Anomalous thrust production from an RF test device measured on a low-thrust torsion pendulum. 50th AIAA/ASME/SAE/ASEE Joint Propulsion conference. Fetta, G.P., 2014. Numerical and experimental results for a novel propulsion technology requiring no on-board propellent. 50th AIAA/ASME/SAE/ASEE Joint Propulsion Conference. AIAA.

Juan, Y., 2012. Net thrust measurement of propellantless microwave thrusters. Acta Physica Sinica, 61, 11.

McCulloch, M.E., 2007. The Pioneer anomaly as modified inertia. MNRAS, 376,338 .

McCulloch, M.E., 2010. Minimum accelerations from quantised inertia. EPL, 90, 29001.

McCulloch, M.E., 2012. Testing quantised inertia on galactic scales. Astro. \& Space Sci., 342, 575.

McCulloch, M.E., 2015. Can the emdrive be explained by quantised inertia? Progress in Physics, 11, 78-80.

Mullins, J., 2006. Relativity drive: the end of wings and wheels? New Scientist (2568), p30-34.

Shawyer, R, 2008. Microwave propulsion - progress in the emdrive programme. 59th International Astronautical conference. IAC-2008. Glasgow, UK.

Tajmar, M., G. Fiedler, 2015. Direct thrust measurements of an EM drive and evaluation of possible side effects. 51st AIAA/SAE/ASEE Joint Propulsion Conference, Propulsion and Energy Forum, Orlando, Florida.

\section{Figures}

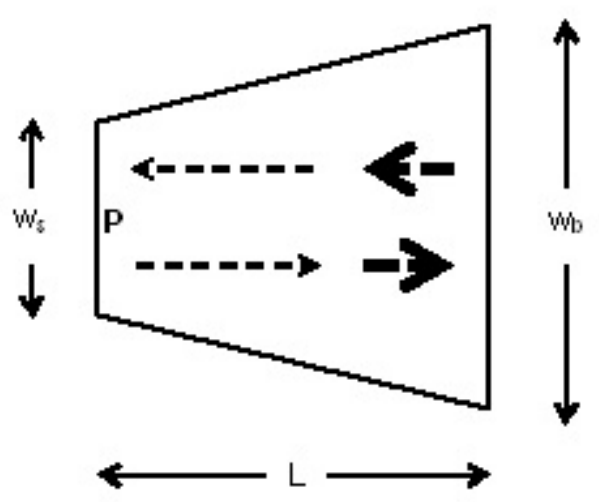

Figure 1. The emdrive cavity (solid line) showing a photon moving right (lower dashed arrows) and then bouncing back towards the left (upper arrows). The 
photon's mass is shown by line thickness and its speed is shown by the line length. For both rightward and leftward moving photons the mass change caused by $\mathrm{MiHsC}$ violates the conservation of momentum, which can only be satisfied by, in both cases, a force acting towards the narrow end, changing its speed. 